\title{
Melatonin MT2 receptor agonist IIK-7 produces antinociception by modulation of ROS and suppression of spinal microglial activation in neuropathic pain rats
}

This article was published in the following Dove Press journal:

Journal of Pain Research

\author{
Yaswanth Kuthati' \\ Venkata Naga \\ Goutham Davuluri \\ Chih-Ping Yang ${ }^{3}$ \\ Hsiao-Cheng Chang' \\ Chih-Peng Chang ${ }^{2}$ \\ Chih Shung Wong ${ }^{1,4}$ \\ 'Department of Anesthesiology, Cathy \\ General Hospital, Taipei, Taiwan; \\ ${ }^{2}$ Department of Microbiology \& \\ Immunology, College of Medicine, \\ National Cheng Kung University, Tainan, \\ Taiwan; ${ }^{3}$ Department of Anesthesiology, \\ Taoyuan Armed Forces General Hospital, \\ Taoyuan, Taiwan; ${ }^{4}$ Graduate Institute of \\ Medical Sciences, National Defense \\ Medical Center, Taipei, Taiwan
}

Correspondence: Chih Shung Wong Department of Anesthesiology, Cathay General Hospital, \#280, Renai Road, Section 4, Taipei, Taiwan

Tel +886022708 2I 21 ext. 35I0

Email w82556@gmail.com
Background: In recent years, several melatonin (MLT) receptor agonists have been approved by FDA for the treatment of sleep disorders and depression. Very few studies have shed light on their efficacy against neuropathic pain (NP). IIK-7 is an MT-2 agonist known to promote sleep. Whether IIK-7 suppresses NP has not been reported, and the signaling profile is unknown.

Objective: To investigate the effect of melatonin type 2 receptor agonist IIK-7 on partial sciatic nerve transection-induced NP in rats and elucidate the underlying molecular mechanisms.

Methods: NP was induced by the PSNT in the left leg of adult male Wistar rats. On posttransection day 7 , rats were implanted with intrathecal (i.t) catheter connected to an infusion pump and divided in to four groups: sham-operated/vehicle, PSNT/vehicle, PSNT/0.5 $\mu \mathrm{g} / \mathrm{hr}$ IIK-7 and PSNT/0.5 $\mu \mathrm{g}$ IIK-7/1 $\mu \mathrm{g}$ 4-p/hr. To test the MT-2 dependence on IIK-7 activity, the animals were implanted with a single i.t catheter and injected MT-2 antagonist 4-Phenyl-2propionamidotetralin (4-p) 20 mins prior to IIK-7 injection on day 7 after PSNT. The antinociceptive response was measured using a mechanical paw withdrawal threshold. Activation of microglial cells and the expression of NP-associated proteins in the spinal cord dorsal horn was assessed by immunofluorescence assay (IFA) and Western blotting (WB). Reactive oxygen species (ROS) scavenging ability of IIK-7 was evaluated by using bone marrow-derived macrophages (BMDM).

Results: Treatment with the MT-2 agonist IIK-7 significantly alleviated PSNT-induced mechanical allodynia and glial activation along with the inhibition of P44/42 MAPK, HMGB-1, STAT3, iNOS and casp-3 proteins.

Conclusion: IIK-7 attenuates NP through the suppression of glial activation and suppression of proteins involved in inflammation and apoptosis. MT-2 receptor agonists may establish a promising and unique therapeutic approach for the treatment of NP.

Keywords: neuropathic pain, MT-2 receptor, IIK-7, allodynia

\section{Introduction}

Neuropathic pain (NP) is a chronic pain condition resulting from damage or disease to the somatosensory system and is difficult to diagnose, with limited treatment options. In addition, modern lifestyles and chronic diseases have significantly increased the number of new cases. Some of the common conditions that are known to damage the somatosensory nervous system include HIV infection, 
chemotherapy, leprosy, diabetes, syphilis, myocardial ischemia, amputation, alcoholism and surgery. ${ }^{1}$ Some of the USFDA-approved first- and second-line treatment options for NP include tricyclic antidepressants and calcium channel $\alpha 2$-delta ligands, with opioids as a last resort for the pain management. ${ }^{2}$ Nevertheless, insufficient pain relief, addiction, tolerance and severe side effects are some of the limitations that necessitate novel therapeutic interventions. Collective evidence has proven antinociceptive effects of MLT and its receptor agonists against nociceptive and NP in preclinical models, and some of the approved MLT agonists for the treatment of sleep disorders are known to have excellent safety and tolerability. ${ }^{3}$

In recent years, several MLT receptor agonists like Ramelteon and Tasimelteon were approved for the treatment of sleep and depressive disorders and several others have entered clinical trials including TIK-301 and Piromelatine. ${ }^{4}$ Although MLT receptor agonists are approved for the treatment of sleep and depressive disorders, none of them have been yet approved for the treatment of NP. However, many promising pre-clinical findings suggest that MLT and its receptor agonists have a strong potential in managing chronic pain conditions. ${ }^{5-9}$

Recent evidence shows that there is a relation between sleep and NP. One recent study concluded that about $80 \%$ of patients suffering from NP have sleep disorders. ${ }^{10}$ It has also been reported that sleep deprivation can greatly reduce the pain thresholds along with signs of depression and anxiety which can aggravate pain. ${ }^{11}$ It is also known that sleep disturbances can greatly increase the risk of developing chronic pain. ${ }^{12}$

Recently, Gobbi et al demonstrated that analgesic properties of MLT are mediated specifically by MT-2 receptors by using MT-2 antagonists. ${ }^{13,14}$ However, the receptorindependent mechanisms underlying the antinociceptive actions of the MT-2 agonists are not explored so far.

IIK-7 is a tetracyclic analog of MLT that specifically binds to MT receptor. ${ }^{15}$ This agonist is known to have 90folds more selectivity toward MT2 receptor $(\mathrm{pKi}=10.30)$ subtype over MT1 (pKi =8.34) with a 6-fold higher affinity toward MT2 receptor subtype than MLT. ${ }^{16}$ IIK-7 is known to promote sleep and protect the cells from electromagnetic stress like MLT. ${ }^{17,18}$ However, to date, there are no reports on the anti-nociceptive properties of this agonist. In the present study, we for the first time assessed the anti-nociceptive effects of the selective MT-2 agonist IIK7 in partial sciatic nerve transection (PSNT) rats, and, we studied the underlying molecular mechanisms, involving glial cells activation and signaling pathways.

\section{Methods}

The protocols used in our study are reviewed and approved by the Animal Care and Use Committee of the National Defense Medical Center and comply with the guidelines given by National Institute of Health Guide for the Care and Use of Laboratory Animals. Male Wistar at 7 weeks of age (BioLASCO Taiwan Co., Ltd., Taiwan) were housed ( 2 per cage) with soft bedding material on a $12 \mathrm{hrs}$ night/day cycle with unrestricted access to food and water. All efforts were taken to minimize the animal number and suffering.

\section{Establishment of the neuropathic pain animal model \& intrathecal catheterization}

PSNT is accomplished according to previously reported procedures. ${ }^{19}$ In the PSNT rats, the left sciatic nerve was carefully exposed till mid-thigh level, and a prolene 7-0 ligature was inserted through the midpoint of the nerve just cranially to the branch running to the musculus biceps femoris, resulting in half of the nerve being transected in the ventrocranial direction up to the ligature. In the sham group, the left sciatic nerve was exposed and then the wound was closed with sutures. From operation day to day 7, the paw withdrawal thresholds are measured in sham-operated rats and PSNT rats to mechanical stimulus. Rats presenting any sign of motor deficit were omitted from the study. On day 7 after PSNT, intrathecal catheter/osmotic pump is implanted under $2-2.5 \%$ isoflurane anesthesia. $^{20,21}$ The intrathecal catheter was constructed as described previously. ${ }^{22}$ The rat's cisternal membrane is carefully punctured to insert a polyethylene catheter (PE 10 tubing, $8.0 \mathrm{~cm}$ ) and threaded caudally to reach the lumbar enlargement of the spinal cord. The rostral end of the catheter was exteriorized at the top of the head, and the wound closed with sutures. IIK-7 (Santa Cruz Biotech, Dallas, Texas) is first dissolved in $4 \%$ DMSO, then diluted with $45 \% \mathrm{w} / \mathrm{v}$ (2-hydroxypropyl)beta-cyclodextrin to improve solubility. To test the dose response, a single catheter was implanted in animals $(n=6)$ and various doses of drug are intrathecally administered through a syringe $(10,100,200,400,500$ and $5000 \mathrm{ng}) 7$ days post PSNT. After drug administration, the catheters were flushed with $15 \mu \mathrm{L}$ saline and 30 mins post-injection the animals are randomly assigned to undergo paw 
withdrawal threshold test. For intrathecal infusion, an osmotic pump (flow rate $1 \mu \mathrm{L} / \mathrm{hr}$; ALZET, Cupertino, California) filled with vehicle (drug diluent), MT-2 agonist IIK-7 $(0.5 \mu \mathrm{g} / \mathrm{hr})$, MT-2 antagonist 4-p(Tocris Biosciences Bristol UK) (1 $\mu \mathrm{g} / \mathrm{hr}) / \mathrm{IIK}-7(0.5 \mu \mathrm{g} / \mathrm{hr})$ (all at $1 / \mu \mathrm{L} / \mathrm{hr}, \mathrm{n}=6$ in each group) was connected to the intrathecal catheter. The pumps were installed subcutaneously, and the wound was closed with silk sutures. Rats that showed neurological deficits after intrathecal catheterization were eliminated from the study. To evaluate the efficacy of the drug or drug in combination with antagonist, the paw withdrawal threshold is measured from day 8 to day 13 after osmotic pump installation.

To test the effect of pretreating antagonist prior to the agonist, a single intrathecal catheter is implanted in rats as described above ( $n=6$ in each group) and injected with 10 $\mu \mathrm{L}$ saline followed by the injection of vehicle, IIK-7 (5 $\mu \mathrm{g}$ / $10 \mu \mathrm{L})$ or $4-\mathrm{p}(10 \mu \mathrm{g} / 10 \mu \mathrm{L})$ followed by a single injection of IIK-7 $(5 \mu \mathrm{g} / 10 \mu \mathrm{L}) 20$ mins post-injection of antagonist. After drug administration, the catheters were flushed with $15 \mu \mathrm{L}$ saline and 30 mins post-injection the animals are randomly assigned to undergo paw withdrawal threshold test from day 8 to day 11 .

\section{Behavior test for tactile allodynia}

In this observer-blinded experiment, the sensitivity of the plantar region was measured using an automatic von Frey Dynamic Plantar Aesthesiometer (Ugo Basile, Comerio, Italy). Rats were individually placed in a plastic cage (25 $\mathrm{cm}$ long $\times 10 \mathrm{~cm}$ wides $\times 14 \mathrm{~cm}$ high) with a wire-meshed floor and acclimatized for 20 mins before each session of testing and then a paw withdrawal response was induced by gradually increasing the force using a blunt-end metal filament $(0.5 \mathrm{~mm})$ fixated on the mid-plantar surface of the hind paw. The force was primarily below the detection threshold, and then was increased from 1 to $50 \mathrm{~g}$ in $1 \mathrm{~g}$ steps over 25 seconds, then maintained at $50 \mathrm{~g}$ for another 10 seconds. The mechanical withdrawal threshold was taken as the force required initiating hind paw reflex and was recorded as the mean of 3 measurements at 2 mins time intervals.

\section{Spinal cord preparation and Western blotting analysis}

After the behavioral testing, all the rats were euthanized by exsanguination under anesthesia with isoflurane (Abbott Laboratories Ltd., Queenborough, Kent, United Kingdom), and the left dorsal quadrant portion of the lumbar spinal cord enlargement was detached and stored at $-80^{\circ} \mathrm{C}$ till usage. The spinal cord samples were homogenized in icecold 1X RIPA lysis buffer (Temecula, CA) using an ultrasonic cell disruptor (Misonix, Inc. USA) and then the homogenate was centrifuged at 13,000 RPM for $30 \mathrm{mins}$ at $4{ }^{\circ} \mathrm{C}$. The supernatant was collected and its protein concentration was determined using the Bradford protein assay. The proteins were denatured by heating at $90^{\circ} \mathrm{C}$ for 10 mins in an equal volume of reducing sample buffer and subjected to separation using $12 \%$ SDS-PAGE followed by protein transfer on to a PVDF membrane (Pall, Ann Arbor, MI, USA) and blocked using 5\% skim milk in TBST (0.05\% Tween 20 in TBS). Primary antibodies against HMGB-1 (Wuhan Fine Biotech, Wuhan, China, Cat \# FNab03924), p44/42 MAPK (Erk1/2) (Cell Signaling Technology, Beverly, MA, USA, Cat \# 9102), STAT3 (Milliopre, USA Cat\# 06-596), iNOS (Thermo Fischer Scientific, MA, USA, Cat\# PA3-030A), casp-3 (Imgenex, Novus Biologicals, Littleton, CO, USA, Cat \# 31A1067), $\beta$-actin (Sigma-Aldrich, St Louis, MO, USA) were incubated at $4{ }^{\circ} \mathrm{C}$ overnight followed by the wash with TBST. HRP-conjugated goat anti-rabbit (Leadgene Biomedical Cat\# 20202) or rabbit anti-mouse IgG secondary antibodies (Leadgene Biomedical Cat\# 20112) (1:5000 dilution) were incubated for another $3 \mathrm{hrs}$ and detected using an Enhanced Chemiluminescence Western Blotting Kit (Advansta, Menlo Park, CA, USA).

\section{Bone-marrow-derived cell culture and ROS evaluation}

Murine bone-marrow-derived macrophages (BMDMs) were generated by flushing bone marrow cells from femurs and tibias of $6-10$-week-old BALB/c mice. These cells were cultured for 5-6 days in RPMI (Gibco) containing $10 \%$ FBS and $10 \mathrm{ng} / \mathrm{mL}$ recombinant mouse M-CSF (Peprotech, Rocky Hill, NJ, USA). To check the ROS scavenging ability of IIK-7, the cultured BMDM cells $4 \times 10^{5}$ cells are seeded in 6-well plates and divided into four groups. The first group is left without any treatment (Mock), the second group is treated with IIK-7 (25 ng) and the third group is treated with PAM3CSK $4(5 \mu \mathrm{g} / \mathrm{mL})$. The fourth group is pre-incubated for 30 mins with IIK-7 followed by the addition of PAM3CSK4 with additional incubation for $12 \mathrm{hrs}$. Finally, DCFHDA is added to all four groups and readings are obtained at FL-1 channel using a flow cytometer. 


\section{Immunofluorescence assay}

For the spinal cord IF, rats were deeply anesthetized with isoflurane and perfused with $0.15 \mathrm{M}$ phosphate buffer ( $\mathrm{PB}$, $\mathrm{pH}$ 7.2) followed by $4 \%$ paraformaldehyde in $\mathrm{PB}$. The dorsal quadrant of the lumbar spinal cord enlargement was detached and embedded in optimal cutting temperature compound until usage. The samples were sectioned $(10 \mu \mathrm{M})$ and air-dried for 30 mins and first washed with PBS (3x) and then treated with acetone for 2 mins followed by a wash with PBS (3x). The slides were incubated overnight with primary antibody, Alexa Fluor ${ }^{\circledR} 488$ antirat $\mathrm{CD} 11 \mathrm{~b} / \mathrm{c}$ (Biolegend, San Diego, CA, USA, CAT\# 201812) (1:200) and washed with PBS (3x) followed by the addition of secondary antibody (1:100 dilution) and DAPI. The slides were imaged using a fluorescence microscope.

\section{Statistical analysis}

Two-way mixed ANOVA analysis was used to study the effect of time on the different drug-treated groups, as well as interaction between time and groups. Two-way repeated measures ANOVA was used for the analyses of main effect of time, main effect of paws and interactions between time and paws. Withdrawal threshold data were converted to maximal possible effect (\%MPE), according to the formula: \%MPE $=[$ (postdrug threshold-post-injured baseline threshold)/(cutoff threshold-post-injured baseline threshold)] $\times 100$. One-way ANOVA was used to detect the group differences where no time course was involved (eg, in vitro test in BMDM for ROS scavenging). Bonferroni post hoc (for all comparisons) and
Bonferroni's multiple comparison tests (for making a restricted set of comparisons) were performed for all pairwise comparisons. GraphPad Prism version 5.01 (GraphPad Software, San Diego, CA) was used to analyze the data. $P<0.001$ was considered statistically significant. Figures were generated using GraphPad Prismversion 5.01 (GraphPad Software, San Diego, CA). Data are expressed as mean \pm S.E.M.

\section{Results}

\section{IIK-7 attenuates sciatic nerve-transection- induced pain}

The anti-nociceptive effect of intrathecal MT-2 agonist IIK-7 was examined in the PSNT rats. After baseline measurement for seven days, IIK-7 was intrathecally administered (10, 100, 200, 400, 500 and $5000 \mathrm{ng}$ ), and then the PWT was measured after 20 mins. IIK-7 dosedependently reversed the mechanical allodynia induced by PSNT. The ED50 of intrathecal IIK-7 is $342 \mathrm{ng}$ (Figure S1). Figure 1 shows the temporal changes of left hind paw withdrawal to the plantar metal filament during infusion of drugs/saline (say 7 to day 13). There was no significant difference in the baseline of paw withdrawal threshold (PWT) in rats prior to nerve transection (depicted as day -1 in Figure 1). In the nerve-transected groups, mechanical allodynia developed on the first day and the withdrawal threshold remained stable from $3 \mathrm{rd}$ day after nerve transection till day 7 (Figure 1). The mean withdrawal threshold pre-operation was (mean \pm SD) 40 $\pm 2.0 \mathrm{~g}$ and decreased progressively to $20 \pm 2.0 \mathrm{~g}$, on day 7 after PSNT. In contrast, PWT of transection/IIK-7,

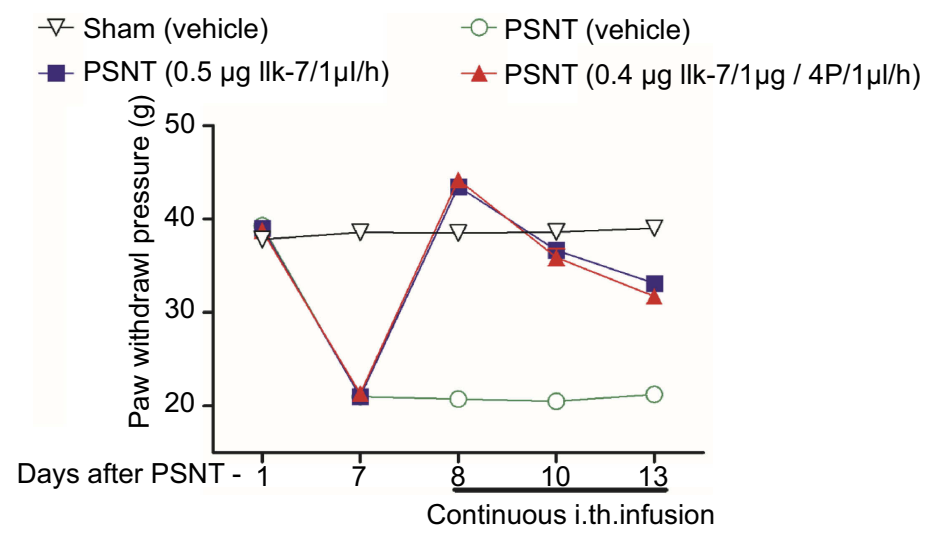

Figure I Continuous intrathecal infusion of melatonin type-2 (MT-2) agonist IIK-7 attenuated the development of nerve-transection-induced neuropathic pain ( $\mathrm{n}=6$ ). Effects of intrathecal infusion of $0.5 \mu \mathrm{g}$ IIK-7/I $\mu \mathrm{L} / \mathrm{hr}, 0.5 \mu \mathrm{g}$ IIK-7/I $\mu \mathrm{g}+4-$ Phenyl-2-propionamidotetralin $(4-\mathrm{p}) / / \mu \mathrm{l} / \mathrm{hr}$ and vehicle// $\mu \mathrm{L} / \mathrm{hr}$ on the mechanical paw withdrawal threshold in ipsilateral paw of partial sciatic nerve-transected rats and sham. PSNT, partial sciatic nerve transection; IIK-7, N-Butanoyl 2-(9-methoxy-6H-iso-indolo[2, I-a] indol- | I-yl)-ethan-amine; 4-p, 4-Phenyl-2-propionamidotetralin. 
IIK-7-4-p group were significantly higher than those of the transection/vehicle group on all day's post-surgery (day 8 to day13 in Figure 1). On 6th day post-surgery (Day 13), the PWT of ligation/IIK-7, IIK-7-4-p group were $33.1 \mathrm{~g}$ and $31.7 \mathrm{~g}$, significantly higher than those of the transected/vehicle group (21.2 g) (Figure 1). Additionally, we examined if 4-p continuous infusion had any effect on the nociceptive response; we found no significant difference from that of control PSNT groups (data not shown). Next, we further explored, if pre-treatment of rats with a single injection of MT-2 antagonist 4-p can suppress the anti-nociception of IIK-7 (Figure 2). The analgesic effects of IIK-7 were not antagonized reversed when co-infused or pre-treated with MT-2 antagonist 4-p for four days prior to IIK-7 single injection, respectively (Figures 1 and 2). At a tested dose of 0.1-5 $\mu \mathrm{g}$, melatonin alone did not display any anti-allodynic effects (data not shown).

\section{IIK-7 inhibited the expression of $\mathrm{P} 44 / 42$ MAPK, HMGB-I and iNOS expression induced by partial sciatic nerve transection}

To determine the expression of $\mathrm{p} 44 / 42$ MAPK, HMGB1 and iNOS in PSNT group and IIK-7-infused group, the spinal cord protein lysates were analyzed by Western blotting (Figure 3). The results showed that the levels of 44/42 MAPK, HMGB-1 and iNOS proteins were significantly higher in PSNT group in comparison to the Sham group. The expression of $\mathrm{p} 44 / 42$ MAPK, HMGB-1 and iNOS significantly decreased in the IIK-7 infused group

\section{IIK-7 scavenge ROS generated in bone- marrow-derived macrophages in response to TL2 receptor ligand PAM3CSK4}

The anti-oxidant properties of IIK-7 are examined in bonemarrow-derived macrophages (BMDM). The ROS generated in BMDM is quantified following the treatment with Toll-like receptor-2 (TLR2) ligand PAM3CSK4 and finally treated with redox-sensitive dye DCFDA. Significant elevation of cellular ROS $(P<0.05)$ is observed in BMDM treated with PAM3CSK4 as compared to untreated controls Mock and IIK-7 (Figure 4). Pre-treating BMDM 30 mins with IIK-7 significantly inhibited the elevation of ROS levels on treatment with PAM3CSK4, indicating the ROS scavenging capabilities in PAM3CSK4-treated cells thereby maintaining a redox-environment comparable to that of mock and IIK-7-alone treated macrophages (Figure 4).

\section{IIK-7 attenuates nerve-transection- evoked microglia activation and iNOS expression}

In the dorsal horn of the spinal cord of vehicle-infused PSNT rats, ameboid shape (active, OX-42 cells) microglia with a strong green fluorescence was found (Figure 5A). In contrast, in the animals infused with IIK-7, the morphology of the microglia remains in a ramified shape (inactive form) with a very weak fluorescence. Likewise, the expression of iNOS was significantly high in PSNT rats infused with the vehicle in comparison with IIK-7 infused groups (Figure 6A). The iNOS immunoreaction intensity was significantly reduced in IIK-7-treated rats, showing very rapid effect on iNOS activation (Figure 6B).

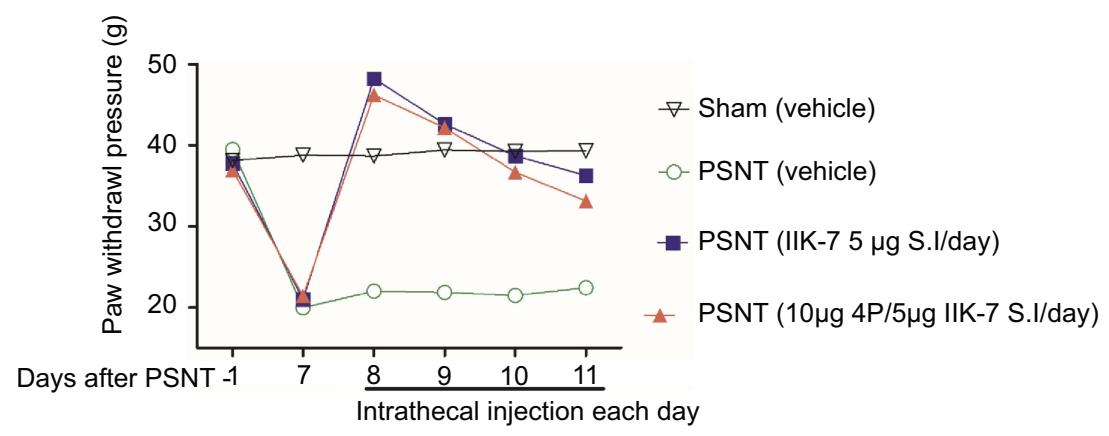

Figure 2 Pre-treatment of IIK-7 antagonist 4-p 20 mins prior to IIK-7 administration did not result in significant blockade of IIK-7-induced anti-nociception in nervetransection-induced neuropathic pain $(\mathrm{n}=6)$. Effects of intrathecal single injection of vehicle $10 \mu \mathrm{L}, 5 \mu \mathrm{g} I \mathrm{KK}-7$ in $10 \mu \mathrm{L}$ vehicle, pretreatment with I0 $\mu \mathrm{g} 4-\mathrm{p}$ in vehicle $10 \mu \mathrm{L}$ followed by the single injection of $5 \mu \mathrm{g}$ IIK-7, on the mechanical paw withdrawal threshold in ipsilateral paw of partial sciatic nerve-transected rats and sham group; $n=6$ rats per group.

Abbreviations: PSNT, partial sciatic nerve transection; IIK-7, N-Butanoyl 2-(9-methoxy-6H-iso-indolo[2, I-a]indol-II -yl)-ethan-amine; 4-p, 4-Phenyl-2-propionamidotetralin. 


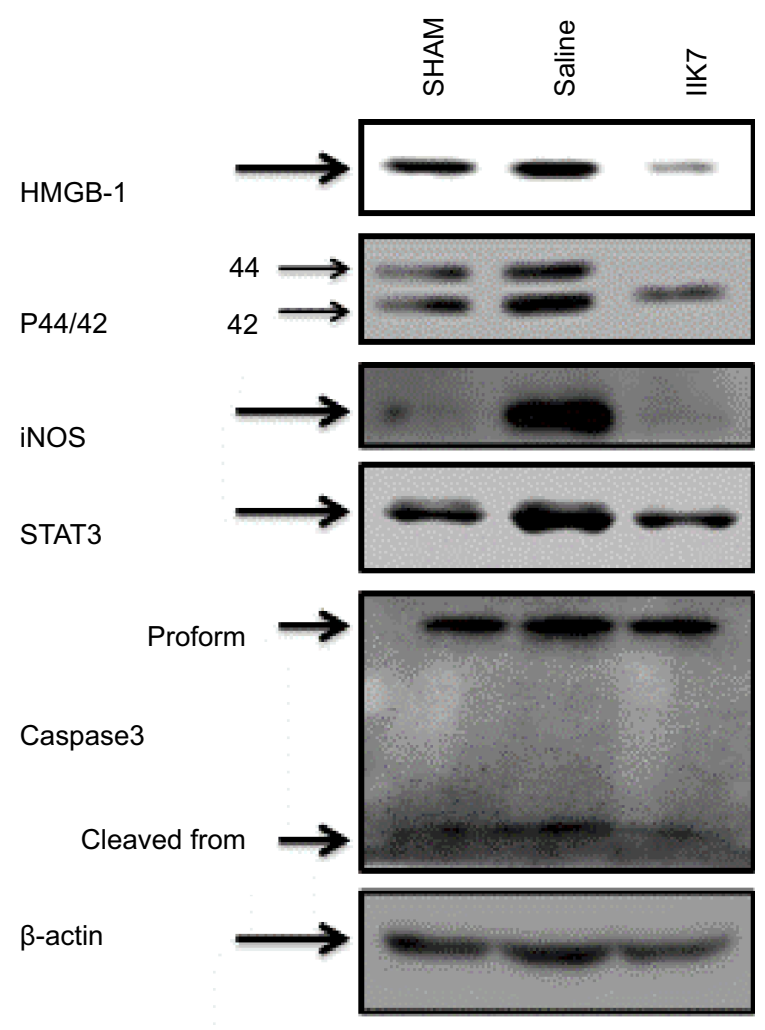

Figure 3 Continuous infusion of IIK-7 attenuated PSNT-induced activation of HMGB-I, PERKI/2, iNOS, STAT3 and caspase-3 in vivo. Representative immunoblots are HMGB-I, P44/42 MAPK, iNOS, STAT 3 and casp-3 from the left dorsal quadrant portion of the lumbar spinal cord lysate of sham surgery or PSNT rats continuously infused with either vehicle or IIK-7 for 7 days using an osmotic pump. Abbreviations: IIK-7, N-Butanoyl 2-(9-methoxy-6H-iso-indolo[2, I-a]indol-I I-yl)ethan-amine; P44/42 MAPK, P44/42 mitogen-activated protein kinase; iNOS, Inducible nitric oxide synthase; STAT3, Signal transducer and activator of transcription 3; CASP-3, caspase3; HMGB-I, High mobility group box I.

\section{Treatment with IIK-7-attenuated PSNT- induced expression of STAT3 and cleaved casp-3}

We further investigated the effect of PSNT on STAT3 and cleaved casp-3 expression. It is found that PSNT persistently increased the expression of STAT3 along with cleaved casp-3, an indicator for the initiation of apoptotic signaling. The PSNT rats infused with IIK-7 significantly decreased the expression of STAT3 and cleaved casp-3 suggesting that IIK-7 could suppress the immune responses in PSNT rats (Figure 3).

\section{Discussion}

In the present study, for the first time, we show that intrathecal infusion of the MT-2 receptor agonist IIK-7 relieved mechanical allodynia in hind paws of PSNT male Wistar rats at an ultra-low dose of $0.5 \mu \mathrm{g} / \mathrm{hr}$ (Figure 1). PSNT resulted in extreme sensitivity to mechanical stimulus as

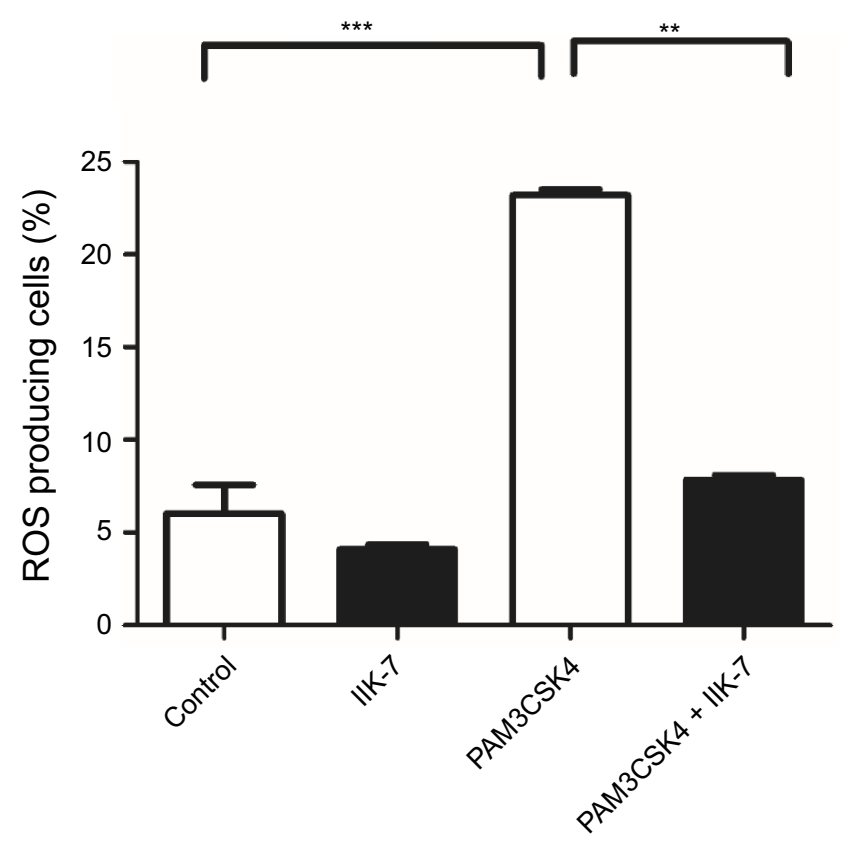

Figure 4 IIK-7 reduces ROS production induced by TLRI/2 agonist PAM3CSK4. The bone-derived macrophages are either untreated (Blank) or treated with IIK-7 (25 ng) or stimulated with triacylated bacterial lipopeptide PAM3CSK $4(5 \mu \mathrm{g} / \mathrm{mL})$ or pre-incubated IIK-7 for 30 mins followed by the addition of PAM3CSK $4(5 \mu \mathrm{g} / \mathrm{mL})$. The cells were further incubated for 12 hrs and readings were noted at FL-I channel using a flow cytometer after the addition of DCFHDA. ROS generation was measured in triplicate. The data shown are the mean \pm SEM values of 3 individual experiments. ${ }^{*} p<0.01$ versus untreated control, *** $p<0.00$ I versus untreated control.

Abbreviations: IIK-7, N-Butanoyl 2-(9-methoxy-6H-iso-indolo[2, I-a]indol- I I-yl)ethan-amine; PAM3CSK4,Pam3CysSerLys4, TLR I/2, Toll-like receptor I/2.

demonstrated by plantar filament tests (Figures 1 and 2). The co-infusion or pre-treatment of PSNT rats with selective MT-2 receptor antagonist 4-p did not significantly inhibit the analgesic potency of IIK-7. Previous studies have shown that pre-treatment with $10 \mu \mathrm{g}$ of MT-2 selective antagonist 4-p, 30 mins prior to MLT administration suppressed MLT-induced increase of paw withdrawal threshold in SNL (sciatic nerve ligation)-induced NP. ${ }^{23}$ In our study $10 \mu \mathrm{g}$ 4-p administration did not show any significant impact on IIK-7's anti-nociception; therefore, it seemed that the anti-nociceptive effect of IIK-7 was irrelevant to MT-2 receptors. However, the receptor-independent free-radical scavenging mechanisms might play a key role in antinociception, and those have been demonstrated previously by several studies on MLT and its agonists. ${ }^{19}$ The receptor-independent mechanisms through which MLT and its agonists expunge the radical scavenging involves the following: 1) through glutathione synthesis, 2) stimulation of antioxidant and inhibition of pro-oxidant enzymes thereby reducing oxidative stress, 3) detoxification of free radicals and maintenance of mitochondria membrane potential. $^{24}$ 
A
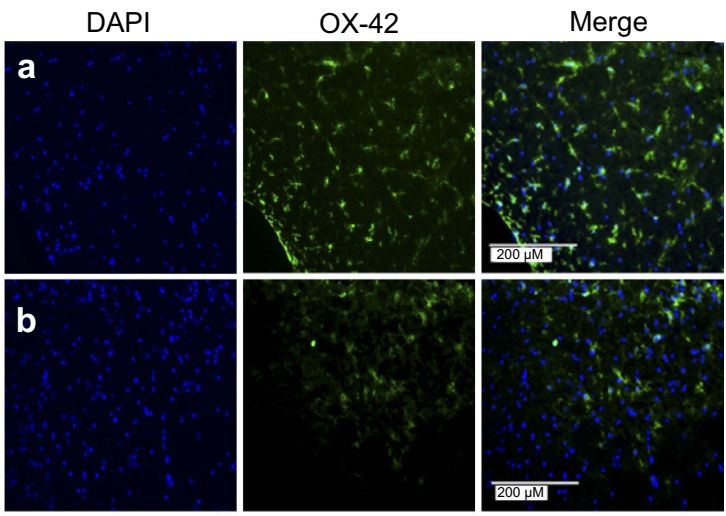

B

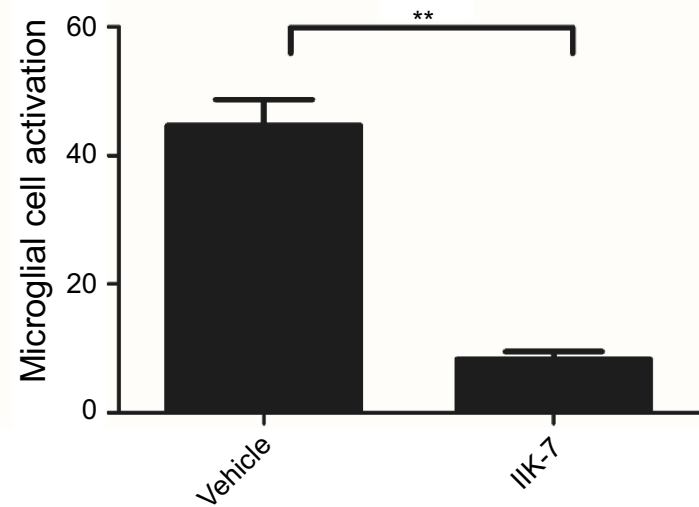

Figure 5 IIK-7 treatment reduces PSNT-induced microglia activation in rat spinal cords. (A) On day 6 after drug treatment, spinal cord sections were fixed and singlelabeled with fluorescein isothiocyanate-labeled anti-OX42 antibodies (green; microglia) and the nuclei stained with 4',6-diamidino-2-phenylindole (DAPI) (blue); then, images were captured and merged by fluorescence microscopy. The sections are from (A) Vehicle-injected PSNT rats, (B) IIK-7-injected rats. The nuclei (blue lane I) and microglia (green lane 2) are merged in lane 3. (B) The microglial activation is quantified by counting the number of activated microglial cells and expressed relative to the value for the vehicle-injected group. Data are analyzed by Tukey test $(* * p<0.01)$. The pictures are representative of those seen in samples from 3 rats. Abbreviations: IIK-7, N-Butanoyl 2-(9-methoxy-6H-iso-indolo[2, I-a]indol- | I-yl)ethan-amine; PSNT, Partial sciatic nerve transection; DAPI, 4',6-diamidino-2phenylindole.

Oxidative stress is considered as one of the most important mechanisms in the development and maintenance of NP. ${ }^{25}$ It can result in the hyperexcitability of nerves in the afferent nociceptors and central neurons triggering spontaneous impulses inside the axons and the DRG-producing NP. The mechanisms through which oxidative stress induces nerve damage or dysfunction include the ROS (Reactive oxygen species) and RNS (Reactive nitrogen species). ROS is known to activate/inhibit several crucial pathways that mediate pain such as activation of P44/42 MAPK, reduction of GABA release, initiation of pro-inflammatory cytokine from the glial cells and inhibition of $\gamma$-aminobutyric acid, which are key pain transmitters (Scheme 1). ${ }^{25,26}$
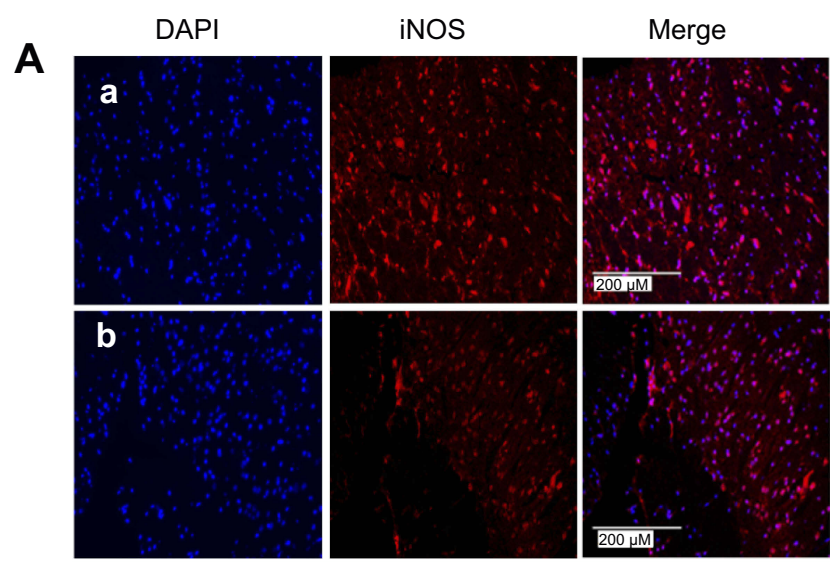

B

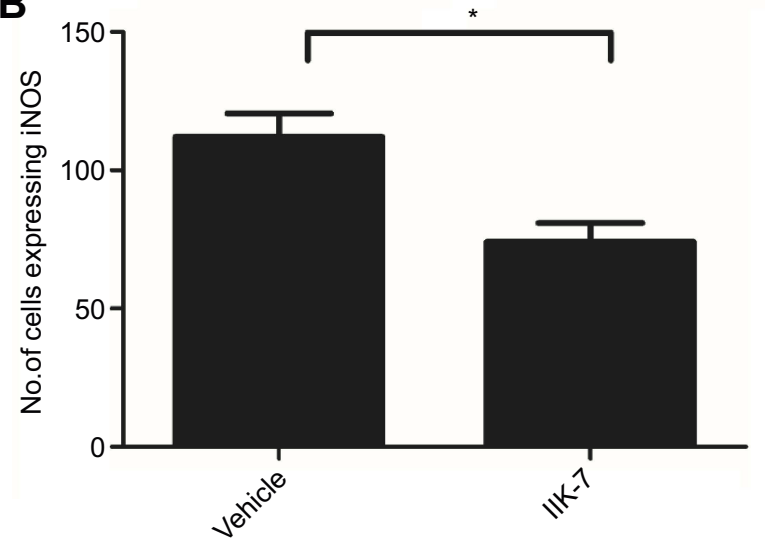

Figure 6 IIK-7 treatment reduces PSNT-induced iNOS expression in rat spinal cords. (A) On day 6 after drug treatment, spinal cord sections were fixed and single-labeled with iNOS rabbit antibody followed by Alexa flour 594 antibody (red) and the nuclei stained with 4',6-diamidino-2-phenylindole (blue), then images were captured and merged by fluorescence microscopy. The sections are from (A) vehicle-injected PSNT rats, (B) IIK-7-injected rats. (B) The iNOS activation is quantified by counting the number of cells expressing iNOS relative to the value for the vehicle-injected group. Data are analyzed by Tukey test $\left({ }^{*} p<0.05\right)$. The pictures are representative of those seen in samples from 3 rats.

Abbreviations: IIK-7, N-Butanoyl 2-(9-methoxy-6H-iso-indolo[2, I-a]indol- I I-yl)ethan-amine; DAPI, 4',6-diamidino-2-phenylindole; iNOS, iNOS Inducible nitric oxide synthase.

Both p44 MAPK (ERK1) and p42 MAPK (ERK2) are distributed ubiquitously in the brain and spinal cord at high levels. Various extracellular stress resulting from nerve damage is known to activate P44/42 MAPK. ${ }^{27}$ Inhibition of ROS is known to downregulate MAPK pathway in various animal models. ${ }^{28}$ MAPK pathway is known to be activated as early as 10 mins post nerve injury in the dorsal horn and $12 \mathrm{hrs}$ post-injury in the microglia through transcriptional modulation of genes involved in central sensitization. ${ }^{29}$ One recent clinical trial has shown that MAPK inhibitor dilmapimod is efficient in relieving NP in patients with good tolerance at higher doses. ${ }^{30}$ To this end, our findings herein show that the IIK-7 blocked 


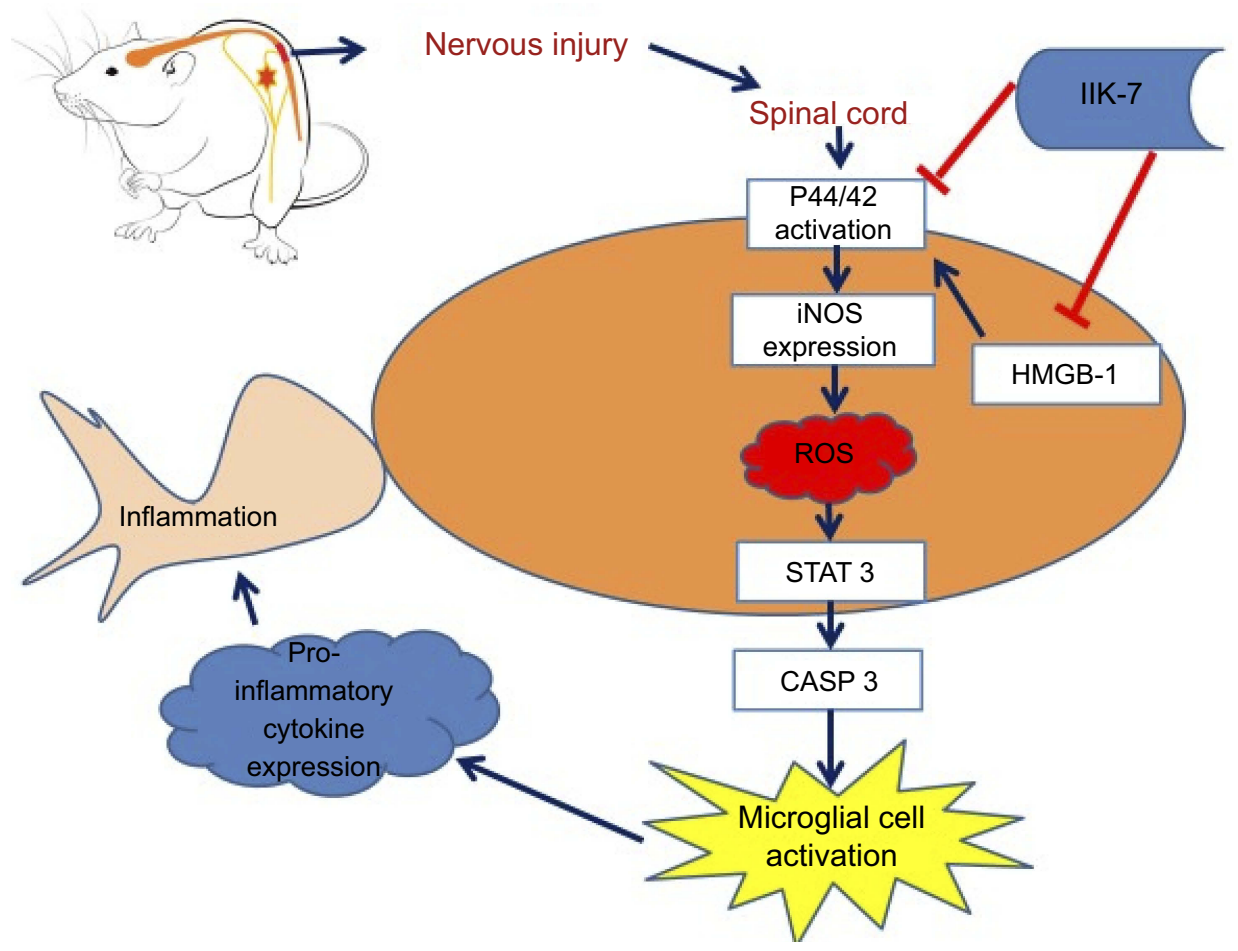

Scheme I Schematic illustration of the potential mechanisms by which N-butanoyl-2-(2-methoxy-6H-isoindolo[2, I-a]indol-I I-yl)ethanamine (IIK-7) modulates pain. The extent of neuroinflammation in neuropathic pain depends on the two-way interactions between neurons and immune cells. According to current literature, microglia are the important cells that mediate neuronal inflammation at the spinal cord level after axotomy. Though microglial are known to be key cells involved in NP, their mechanisms of activation remain elusive. Current evidence indicates that reactive oxygen species (ROS) stimulate the activation. In the present study, we have shown, for the first time, that infusion of IIK-7 prevents microglial cell activation in partial sciatic nerve-transected rats. Simultaneously, IIK-7 treatment affects directly and/or indirectly intracellular pathways, which play a key role in regulating the immune response cascade: STAT3, CASP-3, HMGB-I and iNOS proteins. These molecular actions of IIK-7 might be responsible for its analgesic effect during neuropathic pain and need future investigation.

Abbreviations: IIK-7, N-Butanoyl 2-(9-methoxy-6H-iso-indolo[2, I-a]indol-I I-yl)-ethan-amine; P44/42 MAPK, P44/42 mitogen-activated protein kinase; iNOS, inducible nitric oxide synthase; ROS, reactive oxygen species; STAT3, Signal transducer and activator of transcription 3; CASP-3, caspase3; HMGB-I, High mobility group box I.

PSNT-induced p44/p42 MAPK activation in the dorsal horn of the spinal cord, with the net result being pain relief. p44/42 MAPK activation is known to regulate the expression of several cytokines such as COX-2 and iNOS through transcriptional and translational modifications. ${ }^{31}$

NOS (Nitric oxide synthase) produced during nerve damage can cause nitrosative stress by the production of NO (nitric oxide) and is known to have a significant role in nociception, neuroinflammation and analgesic tolerance, and its inhibition/knockdown is known to alleviate NP. ${ }^{32} \mathrm{NO}$ produced in neurons serves as an important signaling molecule by two isoforms of NOS: neuronal isoform (nNOS) and an inducible isoform (iNOS). Among both, iNOS is known to play a key role in the pathogenesis of neuroinflammation, through the prolonged synthesis of a highly uncontrolled quantity of NO that can be toxic for cells. MLT and its analogs are known to inhibit NOS through receptor-independent mechanisms. ${ }^{33}$ In one recent study, Lin and colleagues have revealed that MLT's effect on NOS is unaffected by MT-2 \& ROR $\alpha$-specific drugs/agonists and further confirmed that MLT can suppress NP receptor-independently by the suppression on NOS. ${ }^{6}$ In the present study, the rats treated with IIK-7 displayed markedly lower levels of iNOS expression compared with sham and PSNT-vehicle-infused rats in both Western blot and immunofluorescence assays (IFAs) (Figures 3 and 6). These results suggest that IIK-7 may attenuate NP by inhibiting iNOS activity. Recent evidence suggests that iNOS expression can potentiate ROS generation in macrophages. ${ }^{34}$

Though emerging evidence suggests the crucial role of ROS in pain, the primary source for ROS generation in NP is still elusive. ROS is produced through various sources in the central nervous system (CNS) including the mitochondria, NADPH oxidase, nitric oxide synthase (NOS), etc. ${ }^{35}$ One recent study by Park et al demonstrated that ROS production is confined to mitochondria of dorsal horn neurons in a rat L5 spinal nerve ligation (SNL) model. ${ }^{36}$ Similarly, intradermal capsaicin injection is shown to induce ROS accumulation primarily in the dorsal horn neurons, indicating the chief role of mitochondrial ROS 
in nociception. ${ }^{37}$ In another study, Gwak et al demonstrated that T10 spinal cord injury can increase the production of ROS in neurons and glial cells in the L4-L5 dorsal horn. ${ }^{38}$ In addition, it was demonstrated that ROS causes NP and locomotor deficit through the activation of neuronal calcium/calmodulin-dependent protein kinase II (CaMKII) by phosphorylation. ${ }^{38}$ Kallenborn-Gerhardt et al demonstrated that knocking out of NOX4, a ROS protein, alleviated nerve-injury-induced NP through the suppression of ROS levels in mice. ${ }^{39}$

Recently, several studies have shown the efficacy of anti-oxidants like phenyl N-tert-Butyl- $\alpha$-phenylnitrone (PBN), 5,5-dimethyl-pyrroline-N-oxide(DMPPO),4-hydroxy2,2,6,6-tetramethylpiperidine-N-oxyl (TEMPOL) and vitamin $\mathrm{A}, \mathrm{C}$ and $\mathrm{E}$ against various NP conditions. ${ }^{40}$ To evaluate the ROS scavenging ability of IIK-7, bone-marrow-derived macrophages are used and ROS generation is triggered using Toll-like receptor-2 (TLR2) ligand PAM3CSK4. TLRs are key initiators of innate response and are triggered upon microbial attack or nerve injury. They are also known to play an important role in microglial activation, inflammation and the development of NP. ${ }^{41}$ The ROS generated upon the treatment of BMDM with TLR2 ligand is detected by redox-sensitive dye DCFDA. Significantly elevated levels of cellular ROS $(P<0.05)$ were observed in BMDM treated with PAM3CSK4 as compared to untreated control and IIK-7-treated cells. Pretreatment with $25 \mathrm{ng}$ IIK-7 followed by treatment with PAM3CSK4 significantly reduced the ROS levels as evident from Figure 4. These results indicate that ROS rapidly accumulates in macrophages treated with PAM3CSK4, while IIK-7 pretreatment can scavenge ROS, and levels remain like those observed for untreated control.

ROS is known to serve as an important trigger for the activation of STAT3 by JAK2-dependent mechanism promoting the expression of inflammation-associated genes. ${ }^{42}$ Studies have also shown that blockade of STAT3 can relieve NP. ${ }^{43}$ In our present study, vehicle-infused PSNT rats showed significant over-expression of STAT-3 when compared to sham groups and intrathecal infusion of IIK-7 prevented the STAT3 activation induced by PSNT (Figure 3). STAT-3-induced apoptosis through caspase activation is one of the well-known mechanisms for neuronal inflammation. ${ }^{44}$

In addition, caspase activation is also known to induce microglial activation and play a pivotal role in the pathogenesis of several neurodegenerative disorders including NP. ${ }^{45}$ Casp-3 activation is one amongst the key indicators for apoptosis as is necessary for protein cleavage. Inhibition of casp-3 is known to suppress NP. ${ }^{46}$ The casp-3 proteins are virtually inactive until they are cleaved by an initiator caspase upon apoptotic signaling and some recent reports suggest that NP increases the cleaved casp-3 levels. ${ }^{47}$ Blocking casp-3 signaling pathway is also known to reduce the apoptotic neurons by the down-regulation of GAP-43 expression, another key regulator of NP. ${ }^{46}$ Our results suggested that treatment with IIK-7 can significantly inhibit cleaved casp-3 (Figure 3) displaying anti-apoptotic properties. Recently, it was demonstrated that Caspases signaling regulates microglial activation through a protein kinase C (PKC)- $\delta$-dependent pathway. ${ }^{48}$

Microglial cells are considered as the gatekeepers of NP and their activation can trigger the release of several neurotransmitters including glutamate and substance $P$, resulting in the release of inflammatory mediators, such as interleukin-11 $\beta$ (IL-1 $\beta$ ), IL-12, ROS, iNOS, Tumor necrosis factor- $\alpha$ (TNF- $\alpha)$, prostaglandin E2, chemokines and adenosine triphosphate (ATP) thereby bringing other immune cells to the injured site and intensifying the inflammatory response. ${ }^{49}$ In one recent study, Kim et al confirmed that NOX-2 ROS production is the crucial step in the induction of NP and demonstrated that ROS is required for pro-inflammatory gene expression in microglial cells. ${ }^{50}$ Blocking microglial activity is an effective strategy in preventing NP. During the resting phase, microglial cells are small with long and thin fine structures; upon activation, they display an amoeboid morphology with large retracted structures. Microglial activation can be detected by OX-42 antibody immunocytochemical staining; vehicle-infused PSNT rat spinal cords in contrast to IIK-7-infused rats displayed enlarged areas of OX42 green immunofluorescence with a distinct morphology indicating their activated state (Figure 5A). The OX42 immunoreaction intensity was significantly reduced in IIK-treated rats (Figure 5B), thus showing a very rapid effect on microglial activation.

HMGB1 is a nuclear protein, but upon encountering mild oxidative stress it translocates to the cytoplasm with subsequent activation of autophagy. ${ }^{51}$ Additionally, several studies reported a spontaneous increase of ROS because of HMGB1 release through the expression of iNOS. $^{52}$ Some evidence indicated that effects of HMGB1 in NP are mediated via its activation of toll-like receptor-4. ${ }^{53}$ The release of HMGB1 in the neuronal cells of CNS is believed to 
regulate the endogenous inflammatory components and influence the adjacent neurons and microglial cells. ${ }^{54}$ It has been previously reported that the exogenous administration of HMGB-1 induces excitability of primary afferent neurons contributing to NP after nerve injury. ${ }^{55}$ Inhibition of HMGB-1 is known to relieve hyperalgesia and allodynia after nerve injury. ${ }^{55}$ Our present study has conclusively found that HMGB1 is actively released upon nerve injury induced by PSNT (Figure 3) and infusion of IIK-7 significantly blocked the expression of HMGB-1.

\section{Conclusion}

In conclusion, we present several lines of evidence to show that MLT MT-2 agonist IIK-7 play an important role in modulating nerve transection-induced pain and neuronal inflammation. First, continuous intrathecal infusion of IIK-7 attenuates PSNT-induced mechanical allodynia. Second, the anti-nociceptive action is not antagonized either with co-infusion or pre-treatment with MT-2 antagonist 4-p, suggesting MT-2 receptor-independent mechanism of anti-nociception. The mechanisms underlying the influence of IIK-7 on NP are yet unclear. IIK-7 may inhibit spinal microglial activation and attenuate NP. Further investigation in determining the underlying mechanisms of IIK-7 anti-nociception may be beneficial.

\section{Abbreviations}

BMDM, bone marrow derived macrophages; CNS, central nervous system; IIK-7, N-Butanoyl 2-(9-methoxy-6H-isoindolo [2,1-a] indol-11-yl)-ethan-amine; MLT, melatonin; MT-2, melatonin type-2, NP, neuropathic pain; PSNT, partial sciatic nerve transection; 4-p, 4-Phenyl-2-propionamidotetralin; ROS, reactive oxygen species.

\section{Acknowledgments}

We thank the Ministry of Science and Technology, Taiwan and Cathay General Hospital for the grant support (MOST:106-2314-B-281-003-MY3; CMRI:CGHMR-A10705).

\section{Author contributions}

All authors contributed to data analysis, drafting or revising the article, gave final approval of the version to be published, and agree to be accountable for all aspects of the work.

\section{Disclosure}

The authors report no conflicts of interest in this work.

\section{References}

1. Colvin LA, Dougherty PM. Peripheral neuropathic pain: signs, symptoms, mechanisms, and causes: are they linked? Br J Anaesth. 2014;114(3):361-363. doi:10.1093/bja/aeu323

2. Smith BH, Lee J, Price C, Baranowski AP. Neuropathic pain: a pathway for care developed by the British Pain Society. $\mathrm{Br} J$ Anaesth. 2013;111(1):73-79. doi:10.1093/bja/aet206

3. Kuthati Y, Lin S-H, Chen I-J, Wong C-S. Melatonin and their analogs as a potential use in the management of neuropathic pain. $J$ Formos Med Assoc. 2018;118(8):1177-1186. doi:org/10.1016/j.jfma.2018.09.017

4. Williams WPT, McLin DE, Dressman MA, Neubauer DN. Comparative review of approved melatonin agonists for the treatment of circadian rhythm sleep-wake disorders. Pharmacotherapy. 2016;36 (9):1028-1041. doi:10.1002/phar.1822

5. López-Canul M, Comai S, Domínguez-López S, Granados-Soto V, Gobbi G. Antinociceptive properties of selective MT2 melatonin receptor partial agonists. Eur J Pharmacol. 2015;764:424-432. doi:10.1016/j.ejphar.2015.07.010

6. Lin -J-J, Lin Y, Zhao T-Z, et al. Melatonin suppresses neuropathic pain via MT2-dependent and -independent pathways in dorsal root ganglia neurons of mice. Theranostics. 2017;7(7):2015-2032. doi:10.7150/thno. 19500

7. Danilov A, Kurganova J. Melatonin in chronic pain syndromes. Pain Ther. 2016;5(1):1-17. doi:10.1007/s40122-016-0049-y

8. Zhu C, Xu Y, Duan Y, et al. Exogenous melatonin in the treatment of pain: a systematic review and meta-analysis. Oncotarget. 2017;8 (59):100582-100592. doi:10.18632/oncotarget.21504

9. Uyanikgil Y, Cavusoglu T, Kilıc KD, et al. Useful effects of melatonin in peripheral nerve injury and development of the nervous system. J Brachial Plex Peripher Nerve Inj. 2017;12(1):e1-e6. doi:10.1055/s-0036-1597838

10. Melikoglu MA, Celik A. Does neuropathic pain affect the quality of sleep? Eurasian J Med. 2017;49(1):40-43. doi:10.5152/eurasianj med.2017.16261

11. Sivertsen B, Lallukka T, Petrie KJ, Steingrimsdottir OA, Stubhaug A, Nielsen CS. Sleep and pain sensitivity in adults. Pain. 2015;156 (8):1433-1439. doi:10.1097/j.pain.0000000000000131

12. Castillo RC, MacKenzie EJ, Wegener ST, Bosse MJ. Prevalence of chronic pain seven years following limb threatening lower extremity trauma. Pain. 2006;124(3):321-329. doi:10.1016/j.pain.2006.04.020

13. Gobbi G, Lopez-Canul M, Palazzo E, et al. PT628. First in class melatonin MT2 receptors agonists for neuropathic pain. Int $J$ Neuropsychopharmacol. 2016;19(Suppl 1):30. doi:10.1093/ijnp/ pyw044.628

14. Lopez-Canul M, Palazzo E, Dominguez-Lopez S, et al. Selective melatonin MT2 receptor ligands relieve neuropathic pain through modulation of brainstem descending antinociceptive pathways. Pain. 2015;156(2):305-317. doi:10.1097/01.j.pain.0000460311.715 $72.5 \mathrm{f}$

15. Faust R, Garratt PJ, Jones R, et al. Mapping the melatonin receptor. 6. melatonin agonists and antagonists derived from $6 \mathrm{H}-\mathrm{Isoindolo}[2,1-\mathrm{a}]$ indoles, 5,6-Dihydroindolo[2,1-a]isoquinolines, and 6,7-Dihydro-5Hbenzo[c]azepino[2,1-a]indoles. J Med Chem. 2000;43(6):1050-1061. doi:10.1021/jm980684+

16. Sugden D, Yeh LK, Teh MT. Design of subtype selective melatonin receptor agonists and antagonists. Reprod Nutr Dev. 1999;39(3):335344.

17. Fisher SP, Sugden D. Sleep-promoting action of IIK7, a selective MT2 melatonin receptor agonist in the rat. Neurosci Lett. 2009;457 (2): $93-96$ 
18. Liu -D-D, Ren Z, Yang G, Zhao Q-R, Mei Y-A. Melatonin protects rat cerebellar granule cells against electromagnetic field-induced increases in $\mathrm{Na}(+)$ currents through intracellular $\mathrm{Ca}(2+)$ release. $J$ Cell Mol Med. 2014;18(6):1060-1070. doi:10.1111/jcmm.12250

19. Lindenlaub T, Sommer C. Partial sciatic nerve transection as a model of neuropathic pain: a qualitative and quantitative neuropathological study. Pain. 2000;89(1):97-106.

20. Yaksh TL, Rudy TA. Chronic catheterization of the spinal subarachnoid space. Physiol Behav. 1976;17(6):1031-1036. doi:10.1016/ 0031-9384(76)90029-9

21. Tai YH, Wang YH, Tsai RY, et al. Amitriptyline preserves morphine's antinociceptive effect by regulating the glutamate transporter GLAST and GLT-1 trafficking and excitatory amino acids concentration in morphine-tolerant rats. Pain. 2007;129(3):343-354. doi:10.1016/j. pain.2007.01.031

22. Tai YH, Wang YH, Wang JJ, Tao PL, Tung CS, Wong CS. Amitriptyline suppresses neuroinflammation and up-regulates glutamate transporters in morphine-tolerant rats. Pain. 2006;124(1-2):7786. doi:10.1016/j.pain.2006.03.018

23. Hsieh M-C, Ho Y-C, Lai C-Y, et al. Melatonin impedes Tet1-dependent mGluR5 promoter demethylation to relieve pain. $J$ Pineal Res. 2017;63(4):e12436. doi:10.1111/jpi.12436

24. Reiter RJ, Tan DX, Manchester LC, Pilar Terron M, Flores LJ, Koppisepi S. Medical implications of melatonin: receptor-mediated and receptor-independent actions. Adv Med Sci. 2007;52:11-28.

25. Yowtak J, Lee KY, Kim HY, et al. Reactive oxygen species contribute to neuropathic pain by reducing spinal GABA release. Pain. 2011;152(4):844-852. doi:10.1016/j.pain.2010.12.034

26. Kanterewicz BI, Knapp LT, Klann E. Stimulation of p42 and p44 mitogen-activated protein kinases by reactive oxygen species and nitric oxide in hippocampus. J Neurochem. 1998;70(3):1009-1016. doi:10.1046/j.1471-4159.1998.70031009.x

27. Qu Y-J, Jia L, Zhang X, Wei H, Yue S-W. MAPK pathways are involved in neuropathic pain in rats with chronic compression of the dorsal root ganglion. Evid Based Complement Alternat Med. 2016;2016;6153215.

28. Choi EK, Yeo J-S, Park CY, et al. Inhibition of reactive oxygen species downregulates the MAPK pathway in rat spinal cord after limb ischemia reperfusion injury. Int $J$ Surg. 2015;22:74-78. doi:10.1016/j.ijsu.2015.08.016

29. Zhuang ZY, Gerner P, Woolf CJ, Ji RR. ERK is sequentially activated in neurons, microglia, and astrocytes by spinal nerve ligation and contributes to mechanical allodynia in this neuropathic pain model. Pain. 2005;114(1-2):149-159. doi:10.1016/j.pain.2004.12. 022

30. Anand P, Shenoy R, Palmer JE, et al. Clinical trial of the p38 MAP kinase inhibitor dilmapimod in neuropathic pain following nerve injury. Eur J Pain. 2011;15(10):1040-1048. doi:10.1016/j.ejpain. 2011.04.005

31. Bhat NR, Zhang P, Lee JC, Hogan EL. Extracellular signal-regulated kinase and p38 subgroups of mitogen-activated protein kinases regulate inducible nitric oxide synthase and tumor necrosis factor-alpha gene expression in endotoxin-stimulated primary glial cultures. $J$ Neurosci. 1998;18(5):1633-1641.

32. De Alba J, Clayton NM, Collins SD, Colthup P, Chessell I, Knowles RG. GW274150, a novel and highly selective inhibitor of the inducible isoform of nitric oxide synthase (iNOS), shows analgesic effects in rat models of inflammatory and neuropathic pain. Pain. 2006;120 (1-2):170-181. doi:10.1016/j.pain.2005.10.028

33. Camacho ME, Carrion MD, Lopez-Cara LC, et al. Melatonin synthetic analogs as nitric oxide synthase inhibitors. Mini Rev Med Chem. 2012;12(7):600-617.

34. Zhao K, Huang Z, Lu H, Zhou J, Wei T. Induction of inducible nitric oxide synthase increases the production of reactive oxygen species in RAW264.7 macrophages. Biosci Rep. 2010;30(4):233-241. doi:10. 1042/BSR20090048
35. Galley HF. Oxidative stress and mitochondrial dysfunction in sepsis. Br J Anaesth. 2011;107(1):57-64. doi:10.1093/bja/aer093

36. Park E-S, Gao X, Chung JM, Chung K. Levels of mitochondrial reactive oxygen species increase in rat neuropathic spinal dorsal horn neurons. Neurosci Lett. 2006;391(3):108-111. doi:10.1016/j. neulet.2005.08.055

37. Schwartz ES, Lee I, Chung K, Chung JM. Oxidative stress in the spinal cord is an important contributor in capsaicin-induced mechanical secondary hyperalgesia in mice. Pain. 2008;138(3):514-524. doi:10.1016/j.pain.2008.01.029

38. Gwak YS, Hassler SE, Hulsebosch CE. Reactive oxygen species contribute to neuropathic pain and locomotor dysfunction via activation of CamKII in remote segments following spinal cord contusion injury in rats. Pain. 2013;154(9):1699-1708. doi:10.1016/j. pain.2013.05.018

39. Kallenborn-Gerhardt W, Schroder K, Del Turco D, et al. NADPH oxidase-4 maintains neuropathic pain after peripheral nerve injury. $J$ Neurosci. 2012;32(30):10136-doi:10.1523/JNEUROSCI.6227-11.2012

40. Oyenihi AB, Ayeleso AO, Mukwevho E, Masola B. Antioxidant strategies in the management of diabetic neuropathy. Biomed Res Int. 2015;2015:515042. doi:10.1155/2015/515042

41. Shi XQ, Zekki H, Zhang J. The role of TLR2 in nerve injury-induced neuropathic pain is essentially mediated through macrophages in peripheral inflammatory response. Glia. 2011;59(2):231-241. doi:10.1002/glia.21093

42. Ng IH, Yeap YY, Ong LS, Jans DA, Bogoyevitch MA. Oxidative stress impairs multiple regulatory events to drive persistent cytokinestimulated STAT3 phosphorylation. Biochim Biophys Acta. 2014;1843(3):483-494. doi:10.1016/j.bbamcr.2013.11.015

43. Liu X, Tian Y, Lu N, Gin T, Cheng CH, Chan MT. Stat3 inhibition attenuates mechanical allodynia through transcriptional regulation of chemokine expression in spinal astrocytes. PLoS One. 2013;8(10): e75804. doi:10.1371/journal.pone.0075804

44. Wan J, Fu AKY, Ip FCF, et al. Tyk2/STAT3 signaling mediates $\beta$-amyloid-induced neuronal cell death: implications in Alzheimer's disease. J Neurosci. 2010;30(20):6873-6881. doi:10.1523/JNEUROSCI.0519-10.2010

45. Joseph EK, Levine JD. Caspase signalling in neuropathic and inflammatory pain in the rat. Eur $J$ Neurosci. 2004;20(11):2896-2902. doi:10.1111/j.1460-9568.2004.03750.x

46. Wu F, Miao X, Chen J, et al. Down-regulation of GAP-43 by inhibition of caspases-3 in a rat model of neuropathic pain. Int J Clin Exp Pathol. 2012;5(9):948-955.

47. Maione S, Siniscalco D, Galderisi U, et al. Apoptotic genes expression in the lumbar dorsal horn in a model neuropathic pain in rat. Neuroreport. 2002;13(1):101-106. doi:10.1097/00001756-20020121000024

48. Burguillos MA, Deierborg T, Kavanagh E, et al. Caspase signalling controls microglia activation and neurotoxicity. Nature. 2011;472 (7343):319-324. doi:10.1038/nature09788

49. Marchand F, Perretti M, McMahon SB. Role of the immune system in chronic pain. Nat Rev Neurosci. 2005;6(7):521-532. doi:10.1038/ nrn1700

50. Kim D, You B, Jo EK, Han SK, Simon MI, Lee SJ. NADPH oxidase 2derived reactive oxygen species in spinal cord microglia contribute to peripheral nerve injury-induced neuropathic pain. Proc Natl Acad Sci U S A. 2010;107(33):14851-14856. doi:10.1073/pnas.1009926107

51. Tang D, Kang R, Livesey KM, Zeh HJ 3rd, Lotze MT. High mobility group box 1 (HMGB1) activates an autophagic response to oxidative stress. Antioxid Redox Sign. 2011;15(8):2185-2195. doi:10.1089/ ars. 2010.3666

52. Sappington PL, Yang R, Yang H, Tracey KJ, Delude RL, Fink MP. HMGB1 B box increases the permeability of Caco-2 enterocytic monolayers and impairs intestinal barrier function in mice. Gastroenterology. 2002;123(3):790-802. doi:10.1053/gast.2002.35391 
53. Kim S, Kim SY, Pribis JP, et al. Signaling of high mobility group box 1 (HMGB1) through toll-like receptor 4 in macrophages requires CD14. Mol Med. 2013;19(1):88-98. doi:10.2119/molmed.2012.00306

54. Kim JB, Sig Choi J, Yu YM, et al. HMGB1, a novel cytokine-like mediator linking acute neuronal death and delayed neuroinflammation in the postischemic brain. $J$ Neurosci. 2006;26(24):6413-6421. doi:10.1523/JNEUROSCI.3815-05.2006
55. Feldman P, Due MR, Ripsch MS, Khanna R, White FA. The persistent release of HMGB1 contributes to tactile hyperalgesia in a rodent model of neuropathic pain. J Neuroinflammation. 2012;9(1):180. doi:10.1186/1742-2094-9-180 


\section{Supplementary material}

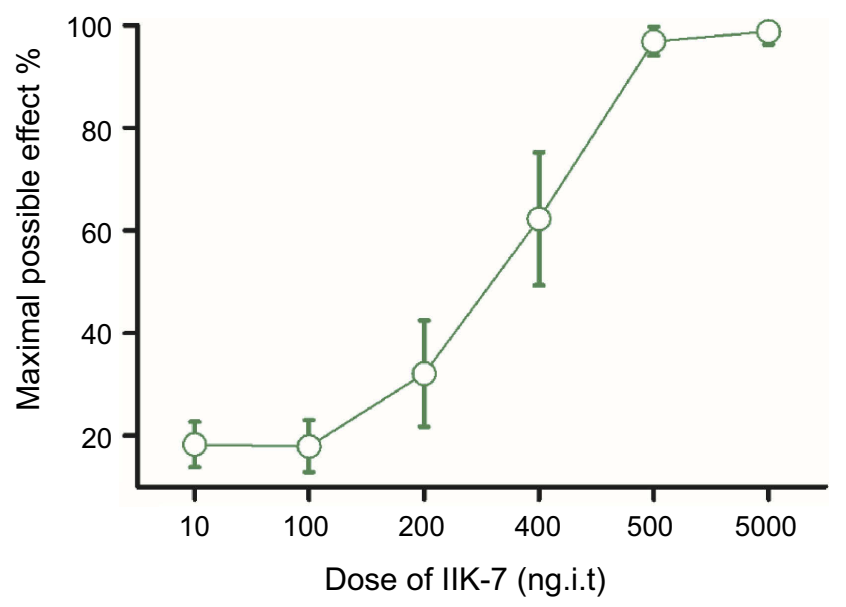

Figure SI Dose-response curve for IIK-7 anti-allodynic effects: ED50 342 ng. IIK-7, N-Butanoyl 2-(9-methoxy-6H-iso-indolo[2, I-a]indol-I I-yl)-ethan-amine; i.t, Intrathecal.

\section{Publish your work in this journal}

The Journal of Pain Research is an international, peer reviewed, open access, online journal that welcomes laboratory and clinical findings in the fields of pain research and the prevention and management of pain. Original research, reviews, symposium reports, hypothesis formation and commentaries are all considered for publication. The manuscript management system is completely online and includes a very quick and fair peer-review system, which is all easy to use. Visit http:// www.dovepress.com/testimonials.php to read real quotes from published authors. 